Rabaska

Revue d'ethnologie de l'Amérique française

\title{
Institut du patrimoine (Université du Québec à Montréal)
}

\section{Yves Bergeron}

Volume 15, 2017

URI : https://id.erudit.org/iderudit/1041169ar

DOI : https://doi.org/10.7202/1041169ar

Aller au sommaire du numéro

Éditeur(s)

Société québécoise d'ethnologie

ISSN

1703-7433 (imprimé)

1916-7350 (numérique)

Découvrir la revue

Citer ce document

Bergeron, Y. (2017). Institut du patrimoine (Université du Québec à Montréal). Rabaska, 15, 328-330. https://doi.org/10.7202/1041169ar d'utilisation que vous pouvez consulter en ligne.

https://apropos.erudit.org/fr/usagers/politique-dutilisation/ 
depuis que le Ministère de la Culture et des communications a coupé la totalité de la subvention au fonctionnement du Centre Mnémo. Nous réussissons à financer des projets qui touchent directement les citoyens de Drummondville, mais l'essentiel de nos activités, qui ont une portée nationale, restent pour le moment impossibles à financer via les programmes existants. Nous espérons fortement que la nouvelle politique culturelle du Québec en cours d'élaboration, nous ouvrira des solutions de financement.

Pierre Chartrand

\section{Institut du patrimoine}

Université du Québec à Montréal

Pavillon AC, local AC-3170

1259 , rue Berri

Montréal (Québec)

Téléphone : (514) 987-3000, poste 2909

H2L 4C7

Courriel : institutdupatrimoine@uqam.ca

Toile : institutpatrimoine.uqam.ca

L'Institut du patrimoine (IP) a été créé en 2004 et regroupe des professeurs, des chargés de cours, des employés, des étudiants et postdoctorants de plusieurs départements, facultés et écoles de l'Université du Québec à Montréal ainsi que des organismes partenaires. L'Institut a pour mission de stimuler la recherche, l'enseignement et la diffusion des connaissances dans tous les domaines reliés au patrimoine, tant matériel qu'immatériel, afin de contribuer au renouveau de la réflexion sur le patrimoine. L'Institut propose de conjuguer les acquis de la recherche et de la formation aux cycles supérieurs en sciences humaines, en arts, et en études urbaines et touristiques, avec la tradition du partenariat avec le milieu tant dans la recherche-action que dans l'offre d'expertise en matière de patrimoine.

L'année 2016-2017 aura été une année de transition pour l'Institut. En 2015, la direction de l'UQÀm s'est engagée dans un processus d'évaluation de ses instituts et il a été résolu de proposer de nouvelles orientations stratégiques pour l'Institut du patrimoine. Le bureau de direction et le conseil scientifique ont proposé un plan d'action qui cible trois grandes orientations pour les trois prochaines années soit de 2017 à 2020.

La première orientation stratégique concerne un projet pédagogique qui oriente l'Institut vers le mandat fondamental de l'Université. Dans cette perspective, il s'agit d'explorer la possibilité de créer une concentration de premier cycle en patrimoine. Ce programme permettrait de suivre des cours dans différents départements. L'UQÀM serait alors la seule université québécoise à offrir une formation dans une approche multidisciplinaire du patrimoine. Ce programme qui reposerait sur des cours existants aura un effet structurant sur le développement de la recherche en patrimoine. Cet objectif permettra de consolider la collaboration des membres de l'IP dans la création de ce programme et de mettre à profit les ressources de l'UQÀM dans toutes les facultés et de développer des formations adaptées pour les organismes en patrimoine. 
Le second objectif stratégique vise à créer deux « bureaux de liaison ». Un premier regroupera des représentants de chacune des composantes du réseau de l'Université du Québec (UQTR, UQO, UQAC, UQAT, UQAR et l'INRS-Urbanisation, culture et société). Il existe dans chaque université du réseau de l'UQ des professeurs chercheurs qui ont développé une expertise en patrimoine et qui apportent leur soutien aux organismes dans leur milieu. Un deuxième « bureau de liaison » sera constitué de représentants des organismes et des milieux professionnels au sein desquels existe également un réseau de chercheurs et de professionnels impliqués dans la conservation et la reconnaissance du patrimoine sous toutes ses formes. Le troisième objectif vise à fédérer à la fois les professeurs de l'UQȦM et des universités partenaires du réseau de l'UQ ainsi que les jeunes chercheurs autour d'un projet collaboratif. Il est proposé de créer un site de veille en patrimoine comme il en existe pour certains secteurs de la culture. L'IP entend intégrer de jeunes chercheurs en patrimoine à ce travail de veille en patrimoine et créer un comité éditorial qui aurait pour mandat de proposer une approche critique de l'actualité en patrimoine en mesurant les impacts et les enjeux de société.

\section{Réalisations}

Bien que le bureau de direction ait convenu en septembre 2016 de se concentrer sur le plan de développement stratégique, l'Institut a maintenu la tenue de la $3^{\text {e }}$ journée thématique de la série « Patrimoine 3.0 » en partenariat avec BANQ. Cette journée d'étude avec pour thème « La culture numérique et l'expérience patrimoniale renouvelée » s'est déroulée dans l'Auditorium de BANQ Vieux-Montréal le 24 février 2017. Les communications sont disponibles en format audio sur le site de l'Institut.

À l'automne, l'Institut s'est associé au Mouvement national des Québécoises et Québécois $(\mathrm{MNQ})$ et plusieurs partenaires pour les « États généraux sur la commémoration » du 6 au 9 octobre 2016. À titre de partenaire, l'Institut a bénéficié d'une excellente visibilité puisque l'évènement était présenté en simultané en ligne. Les communications sont toujours accessibles sur le site du MNQ (mnq.quebec/etatsgeneraux). Le MNQ a publié le «Rapport et les recommandations suite aux États généraux sur les commémorations historiques ».

L'Institut s'est associé à l'Institut du patrimoine culturel de l'Université Laval, le Célat, la Société des musées du Québec et le Musée de la civilisation pour la « journée d'étude sur les musées et la gouvernance stratégique » le 22 février 2017. Cette rencontre au Musée de la civilisation a permis d'accueillir plus de 120 personnes provenant du réseau des musées et du patrimoine. Une seconde journée d'étude sur le même thème a été organisée le 13 juin 2017 à l'Université Paris 3 Sorbonne Nouvelle en collaboration avec l'Association internationale d'études, la Bibliothèque Gaston-Miron de Paris 3 et la Délégation générale du Québec à Paris.

L'Institut s'est également associé avec le programme de doctorat en muséologie, médiation, patrimoine de l'UQÀM et la Société des musées du Québec pour l'organisation d'un colloque sur les « Nouvelles perspectives en muséologie » à l'UQÀM le 25 avril 2017.

$\mathrm{Au}$ chapitre des travaux scientifiques, l'Institut a soutenu financièrement trois ouvrages publiés en 2016. D’abord, Éveil et enracinement. Approches pédagogiques 
innovantes du patrimoine culturel aux Presses de l'Université du Québec sous la direction de Marie-Claude Larouche, Joanne Burgess et Nicolas Beaudry (www. puq.ca/catalogue/livres/eveil-enracinement-3183.html). Puis Yves Bergeron et Julie-Anne Côté ont publié Un nouveau musée pour un Nouveau Monde. Musées et muséologie selon Roland Arpin et «Diriger sans s'excuser». Patrimoine, musée et gouvernance selon Roland Arpin chez L'Harmattan. Le lancement des trois ouvrages a été souligné lors de la $3^{\mathrm{e}}$ journée d'étude sur le patrimoine 3.0 à BANQ le 24 février 2017. Enfin, l'Institut a apporté son soutien à la publication de l'ouvrage électronique ayant pour titre Histoire des musées au Québec. Repères chronologiques (1534-2016) sous la direction de Cyril Simard et Yves Bergeron. L'ouvrage édité par le réseau Économusées et accessible sur le site de la Société des musées du Québec (www. editions-harmattan.fr/index.asp?navig=catalogue\&obj=livre\&no=52263) se veut un projet collaboratif avec la Société des musées du Québec et l'Institut du patrimoine qui prévoit une mise à jour annuelle.

Yves Bergeron 